\title{
Fabrication of hierarchical polycaprolactone/gel scaffolds via combined 3D bioprinting and electrospinning for tissue engineering
}

\author{
Yong-Ze Yu $\cdot$ Lu-Lu Zheng $\cdot$ Hai-Ping Chen • \\ Wei-Hua Chen · Qing-Xi Hu
}

Received: 21 December 2013/Accepted: 14 May 2014/Published online: 10 June 2014

(C) Shanghai University and Springer-Verlag Berlin Heidelberg 2014

\begin{abstract}
It is a severe challenge to construct 3D scaffolds which hold controllable pore structure and similar morphology of the natural extracellular matrix (ECM). In this study, a compound technology is proposed by combining the $3 \mathrm{D}$ bioprinting and electrospinning process to fabricate 3D scaffolds, which are composed by orthogonal array gel microfibers in a grid-like arrangement and intercalated by a nonwoven structure with randomly distributed polycaprolactone (PCL) nanofibers. Human adiposederived stem cells (hASCs) are seeded on the hierarchical scaffold and cultured $21 \mathrm{~d}$ for in vitro study. The results of cells culturing show that the microfibers structure with controlled pores can allow the easy entrance of cells and the efficient diffusion of nutrients, and the nanofiber webs layered in the scaffold can significantly improve initial cell attachment and proliferation. The present work demonstrates that the hierarchical PCL/gel scaffolds consisting of controllable 3D architecture with interconnected pores and biomimetic nanofiber structures resembling the ECM can be designed and fabricated by the combination of $3 \mathrm{D}$ bioprinting and electrospinning to improve biological performance in tissue engineering applications.
\end{abstract}

Keywords Hierarchical scaffold - 3D bioprinting . Electrospinning · Tissue engineering

Y.-Z. Yu ( $\square)$ · L.-L. Zheng · H.-P. Chen · W.-H. Chen •

Q.-X. Hu

Rapid Manufacturing Engineering Center, Shanghai University, Shanghai 200444, People's Republic of China e-mail: yuyz1023@shu.edu.cn

\section{Introduction}

As an interdisciplinary field, tissue engineering applies the principles of engineering and life sciences toward the development of biological substitutes that restore, maintain, or improve tissue function or a whole organ [1-3]. Scaffold, one of the three key elements in tissue engineering, provides temporary support and biomimetic microenvironment for cells attachment, proliferation and differentiation $[4,5]$. The ideal scaffold should have adequate pore size and interconnected porous structures to facilitate cells migration, and allow diffusion of nutrients and metabolic waste products [6, 7]. Moreover, it should also possess the nano-scale features, such as those present in natural ECM, which can enhance cells adhesion and induce contact guidance [8-10]. It is determined not only by the materials but also by the technique to fabricate scaffolds with the above essential properties $[5,11]$.

3D bioprinting technology, as one of the powerful additive manufacturing techniques, has shown the potential to fabricate tissue engineering scaffolds for the repair or regeneration of human tissues and organs [12]. Using this technology, scaffolds are fabricated by extruding materials through the nozzle and depositing strands layer by layer. The main advantage of this technology is that many biomaterials can be used for 3D bioprinting to produce complex structures with precise control of pore size or the spatial distribution of pores by the computer-aided design (CAD) [13-16]. For example, the gel biomaterials can be used to fabricate porous 3D scaffolds under mild conditions by the $3 \mathrm{D}$ bioprinting process without being treated by heat or organic solvents which may influence the biocompatibility of scaffolds [17-19]. However, the pore sizes of scaffolds created by 3D bioprinting are so large that cells will deposit on the bottom of scaffolds. In addition, the 3D 


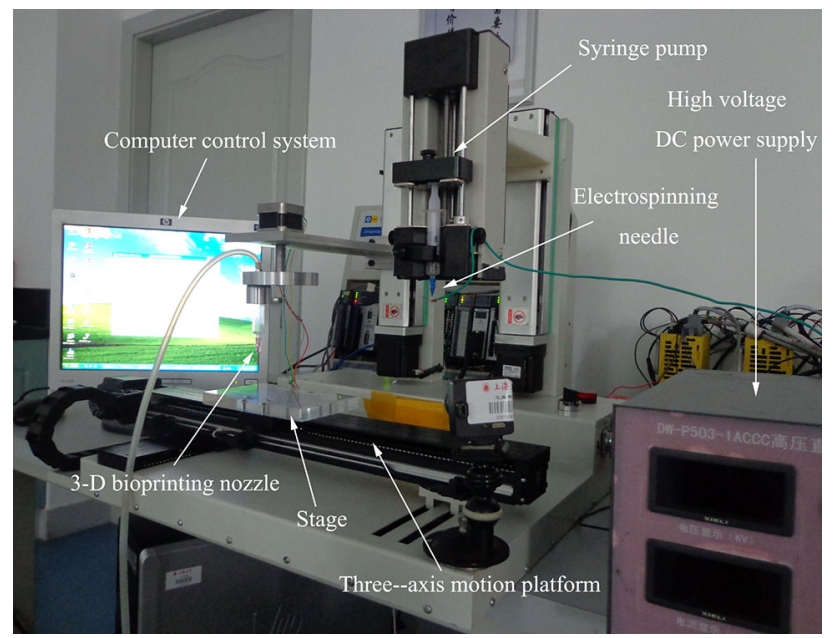

Fig. 1 Prototype of the comprehensive bioprinting system for fabrication of hierarchical scaffolds

bioprinting process cannot fabricate nano-scale fibers as present in natural ECM that have been suggested to enhance initial cells attachment and proliferation [20-22]. Therefore, an improved method is necessary to overcome these problems.

A strategy to overcome these limitations is the combination of the $3 \mathrm{D}$ bioprinting technology with other techniques to create the $3 \mathrm{D}$ scaffolds with controlled pore structures and nano-scale features. Because of the nanofiber sizes and similar morphology to the natural ECM, the nanofibrous scaffolds obtained through electrospinning have received more attention in tissue engineering [23-25]. Thus, in this paper, we introduce a compound technology that combines the 3D bioprinting technology and electrospinning process to fabricate a hierarchical 3D scaffold not only with perpendicular gel microfibers at controllable structural but also the PCL nanofibers ECM-like web embedded in successive layers to enhance initial cells attachment. The microstructure characteristics of hierarchical scaffold are analyzed by scanning electron microscopy (SEM). Moreover, the human adipose-derived stem cells (hASCs) are selected to observe the effects of electrospun nanofibers between microfibers on the cell adhesion and proliferation in the hierarchical PCL/gel scaffolds.

\section{Experimental}

\subsection{Materials}

The PCL pellets with an average molecular weight of 80,000 were obtained from Sigma-Aldrich (Shanghai, China). Dichloromethane, gelatin and sodium alginate were purchased from Sinopharm Chemical Reagent Co.,
Ltd (Shanghai, China). The PCL solution with concentration of $8 \mathrm{wt} \%$ was prepared by dissolving in dichloromethane and then stirred $2 \mathrm{~h}$ by magnetic stirrer at $800 \mathrm{r} / \mathrm{min}$ and $25^{\circ} \mathrm{C}$ for electrospun nanofibers fabrication. To fabricate micro-scale gel strands, $20 \mathrm{wt} \%$ gelatin solution and $4 \mathrm{wt} \%$ sodium alginate solution were blended homogeneously at 1:1 (wt\%) to make the gel.

\subsection{Construction of the comprehensive bioprinting system}

To build the hierarchical scaffolds, a novel comprehensive bioprinting system was developed by combining an electrospinning apparatus into the 3D bioprinting platform. Figure 1 shows the comprehensive bioprinting system for fabrication of hierarchical scaffolds.

The 3D bioprinting platform consisted of a nozzle deposition system, three-axis motion platform, stage, air pump and a computer control system. By processing the designed scaffold model from CAD and converting the data into tool path layer by layer, the computer control system that required input of the processing parameters controlled the pressure, move speed and position. The nozzle and stage were mounted on the three-axis motion platform which was controlled by computer to fabricate the scaffold. The air pump was linked to the nozzle to supply the air pressure for the material delivery.

In this comprehensive system, the electrospinning apparatus was composed of a syringe with metallic needle, a syringe pump and a high voltage DC power supply. The syringe pump and power supply were modulated by the same computer control system.

\subsection{Scaffold design and fabrication}

As shown in Fig. 2, a cubical hierarchical scaffold (the dimension of $20 \mathrm{~mm} \times 20 \mathrm{~mm}$ and 16 layers) which consisted of orthogonal array gel microfibers and electrospun PCL nonwovens was designed. The micro-scale stands were regularly arrangement with the equal distance of adjacent stands in $0.45 \mathrm{~mm}$ during each layer, and the orientations of two successive layers were changed $90^{\circ}$ to form the 3D scaffold with rectangular channels. After fabricating every 2 layers microfibers, a nanofibers web was electrospun and deposited on the layer. Then, a new gel layer was printed and adhered to the electrospun fibers and the previously printed layer.

The 3D bioprinting system with a nozzle (inner diameter of $0.5 \mathrm{~mm}$ ) was used to print the 3D gel scaffold layer by layer. First, the bio-gel as prepared previously was transferred to cartridge of the dispenser. The relative humidity and ambient temperature of the bioprinting system were fixed at $55 \%$ and $25{ }^{\circ} \mathrm{C}$, respectively. The speed range of the stage was $20 \mathrm{~mm} / \mathrm{s}$. Then, the bio-gel material was drawn by 


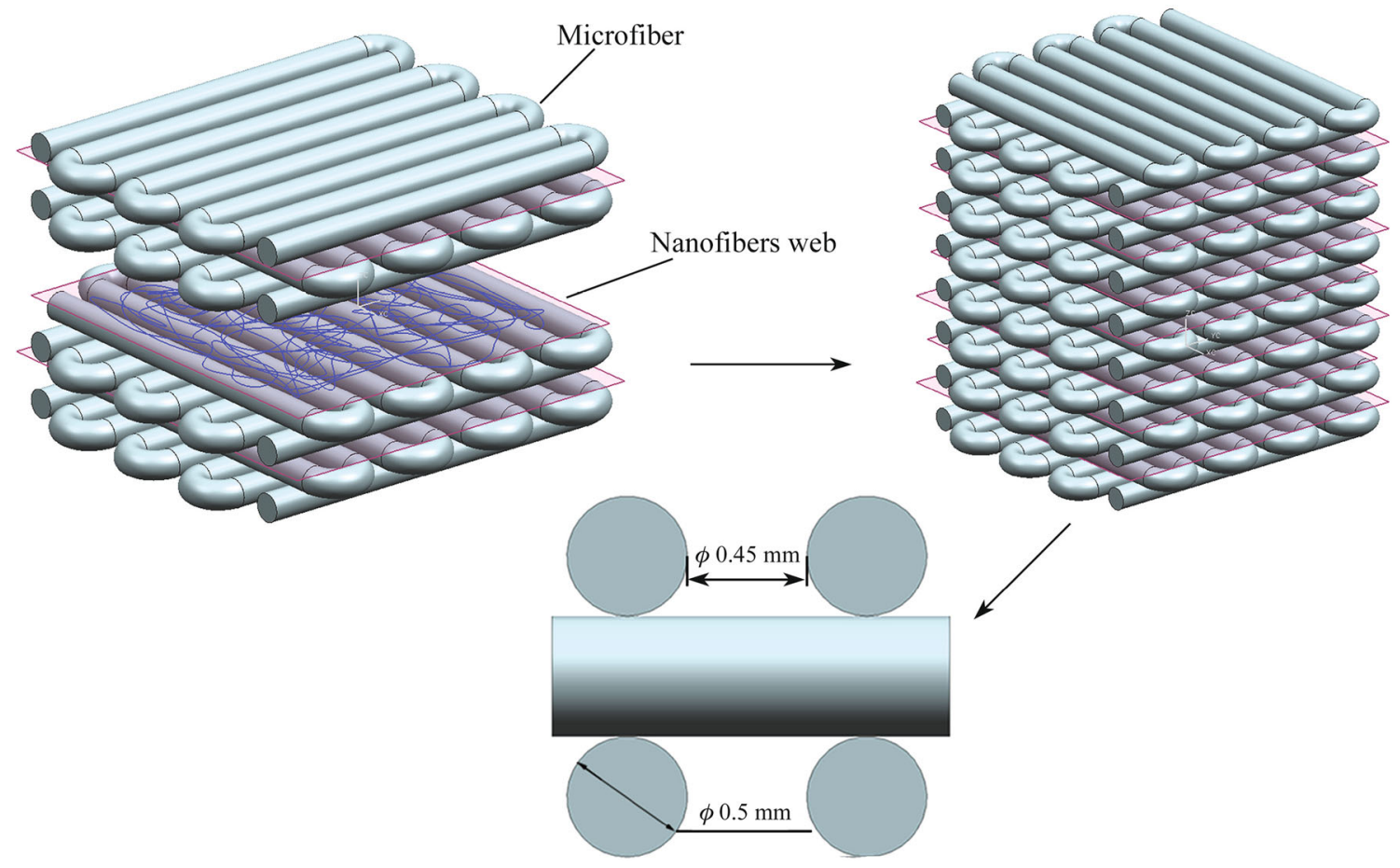

Fig. 2 CAD model of hierarchical PCL/gel scaffold

controlling the pneumatic pressure $((0.35 \pm 0.02) \mathrm{MPa})$, which was supplied by an air pump (ZBM-0.15, Taizhou). The micro-scale gel strands were plotted in layers.

The layers of PCL nanofibers were electrospun by the electrospinning platform of the comprehensive system. The PCL solution was delivered through the metallic needle (inner diameter of $0.32 \mathrm{~mm}$ ) at a flow rate of $0.04 \mathrm{~mL} / \mathrm{min}$. The diameter of nanofibers and pore size of web could be controlled by adjusting the processing parameters. The voltage applied to the needle was fixed at $15 \mathrm{kV}$, and the distance between the tip of needle and collector was $15 \mathrm{~cm}$. The deposition time of electrospinning was $3 \mathrm{~s}$ to fabricate the nanofibers web with bigger pore sizes for cells penetration inside the scaffold [26].

After the completion of compound production process, the scaffold was freeze dried by vacuum freeze dryer (LGJ-10D, Beijing Four-Ring Science Instrument Plant Co. Ltd, China) at $-35{ }^{\circ} \mathrm{C}$ for $48 \mathrm{~h}$ to remove the solvent and water, and then the final hierarchical scaffolds fabricated by $3 \mathrm{D}$ bioprinting technology and electrospinning process were obtained.

\subsection{Scaffold characterizations}

The morphology of the hierarchical scaffold was observed by digital camera (IXUS 125 HS, Canon, Japan), and the micromorphology of electrospun PCL nanofibers and gel microfibers were characterized by the optical microscope (80I, EVERGRAND INTERNATIONAL, Japan) and SEM
(Hitachi SU-1500, Japan). The SEM images were obtained with the acceleration voltage of $10 \mathrm{kV}$.

The porosity of scaffold was calculated according to the following equation:

$P(\%)=\frac{V_{0}-V}{V_{0}} \times 100 \%$,

where $V_{0}\left(\mathrm{~cm}^{3}\right)$ is the total volume calculated by $L \times W \times H$, and $V$ is the actual volume of the scaffold biomaterial. The total volume $\left(V_{0}\right)$ of scaffold was measured by vernier caliper and the actual volume of scaffold was estimated according to a liquid displacement method [27].

\subsection{Cell culture}

For evaluation cells attachment, growth and proliferation on the hierarchical scaffold, human adipose-derived stem cells (hASCs) were seeded onto the hierarchical PCL/gel scaffolds and 3D bioprinting scaffolds (without electrospun nanofibers web) to make a comparison. Cells viability and extracellular matrix secretion were tested over a period of 21 d cultivation.

The hASCs were obtained from National Tissue Engineering Research Center (NTES, Shanghai, China) and cultured in Dulbecco's modified Eagle's medium (DMEM) containing $10 \%$ fetal calf serum, $1 \%$ mixture of $100 \mathrm{U} / \mathrm{mL}$ penicillin and $100 \mu \mathrm{g} / \mathrm{mL}$ streptomycin (all from SigmaAldrich, Shanghai, China) at $37^{\circ} \mathrm{C}$ and in an atmosphere of $5 \% \mathrm{CO}_{2}$. The medium was changed every three days. 
During the period of cells cultured and passage, the cells morphology was observed by using inverted fluorescence microscope (LEICA DMIRE, Leica microsystems Ltd, Germany).

Before seeding the hASCs, the hierarchical and bioprinted scaffold specimens with size of $5 \mathrm{~mm} \times 5 \mathrm{~mm} \times 3 \mathrm{~mm}$ were immersed in $70 \%$ ethanol and exposed to ultraviolet light $1 \mathrm{~h}$ for sterilization, and then placed in culture medium overnight. Then, the cells in passage 3 were seeded on the scaffolds at a density of $2.5 \times 10^{5}$ cells per sample. The cell/scaffold constructs were incubated in $5 \% \mathrm{CO}_{2}$ at $37{ }^{\circ} \mathrm{C}$ for up to $21 \mathrm{~d}$.

\subsection{Evaluation of cell adhesion, morphology and proliferation}

The inversion fluorescence microscope (DM14000 B, LEICA, Germany) was used to observe the initial attachment (1 d) of hASCs on the hierarchical scaffold and bioprinted scaffold. To inspect the detailed morphology of cells on the scaffolds, the cells/scaffold constructs, after incubating for $7 \mathrm{~d}$, were fixed in $2.5 \%$ glutaraldehyde (Sinopharm Chemical Reagent Co., Ltd, Shanghai, China) in phosphate-buffered saline (PBS, Sigma-Aldrich) solution for $30 \mathrm{~min}$ at room temperature and dehydrated in a graded ethanol series. After drying overnight, specimens were sputter-coated with gold, and the morphology of cells cultivated onto the scaffolds was observed by field emission scanning electron microscope (FE-SEM, JSM-6700F, Japan). To investigate the penetration of cells to the thickness direction of the hierarchical scaffold, the confocal laser scanning microscope (CLSM, LSM710, Germany) was used to observe the distribution of cells inside the hierarchical scaffold after $21 \mathrm{~d}$ cell culture.

Cell proliferation on the scaffolds was evaluated by Hoechst 33258 dye for measurement of DNA quantification. Quantification was performed using the Modulus TM PicoGreen dsDNA Assay Kit (YuanPingHao Bio, Beijing, China), according to the manufacturer's instructions, and after the cells cultured in the scaffolds for 1, 4, 7, 14, $21 \mathrm{~d}$ were lysed by osmotic and thermal shock. The intensity of the fluorescence, proportional to the amount of DNA, was measured at excitation wavelength of 360/40 nm and at an emission wavelength of $465 / 40 \mathrm{~nm}$, in a microplate reader (VICTOR X3, PerkinElmer, USA). Triplicates of each sample were tested for a statistical analysis.

\section{Results and discussion}

\subsection{Scaffold fabrication and structural characterization}

In general, the porous architecture, mechanical properties and surface topography play critical roles in tissue regeneration
[28-31]. 3D bioprinting technology can be used to produce scaffolds with reproducible, controllable pore size and strands pitch as a function of pore structure and mechanical properties. Additionally, the ideal scaffolds should have the high surface area and similar morphology of native ECM to significantly enhance initial cells attachment and proliferation. Thus, the electrospun nonwovens can provide a cell entrapment system and nano-scale topographies to promote cells growth.

A hierarchical scaffold, which consisted of gel microfibers and PCL nanofibers web produced by 3D bioprinting and electrospinning respectively, was fabricated. The images of both wet and dry conditions are presented in Fig. 3a, b. As shown in Fig. 3c, the dried scaffold was characterized by a regular and interconnected pore structure which was formed by vertical aligned gel printed microfibers, whose average fiber diameter was $440 \mu \mathrm{m}$ with a standard deviation of $41.19 \mu \mathrm{m}$, and the strand spacing was $(527 \pm 34.38) \mu \mathrm{m}$. Electrospun PCL nanofiber webs were regularly intercalated among every 2 layers of printed microfibers, as shown in Fig. $3 \mathrm{~d}$. The average diameter of nanofiber is $(190 \pm 15.72) \mathrm{nm}$ and the mean pore size is $(20 \pm 8.06) \mu \mathrm{m}$.

\subsection{Porosity test}

There are two kinds of porosity of the hierarchical scaffold: the theoretical porosity $P_{1}(\%)$ of the designed model and the actual porosity $P_{2}(\%)$ of the final drying scaffold. Based on the geometric dimensioning of designed CAD model (see Fig. 2), the theoretical porosity $P_{1}(\%)$ was $60.75 \%$. Because of the moisture sublimation during the vacuum freeze-drying process, the gel microfibers underwent shrinkage to a certain extent and many micropores were left on the surface of strands which may cause a higher porosity. Using the liquid displacement method, we measured the volume of liquid displacement eight times and the actual porosity $P_{2}(\%)$ was $(64 \pm 3) \%$. Freeze dried-induced surface contraction and micropores in gel microfibers did not increase porosity much higher, which might result from the extrusion die swell of gel during the printing process. The porosity rose due to the moisture sublimation and strands contraction, partly offset by diameter increase caused by gel extrusion die swell.

\subsection{Cell culture}

To evaluate the superiority of the hierarchical scaffold consisted of microfibers and nanofibers web for cells culture in tissue engineering, hASCs were seeded and cultivated on both the normal 3D bioprinting scaffold and the hierarchical 3D scaffold, and the comparisons of their attachment, morphology and proliferation were observed. 


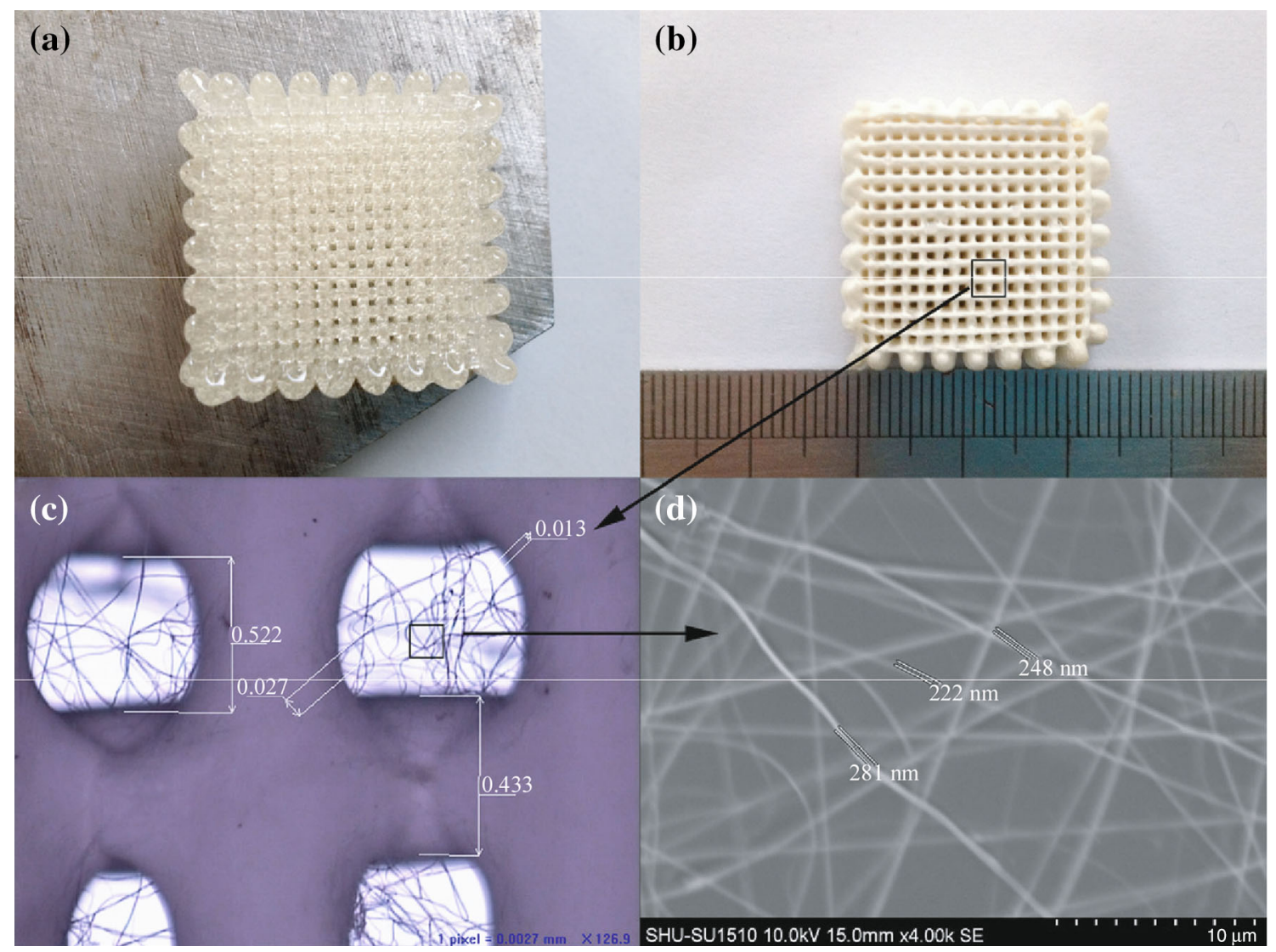

Fig. 3 Photographs of the hierarchical PCL/gel scaffold: a scaffold is shown in wet after fabricating via compound technology; b scaffold is shown in dry after freeze-drying; c micrograph of the hierarchical scaffold; d SEM micrograph of PCL nanofibers

Figure 4 shows the initial attachment of hASCs seeded on the hierarchical scaffold and printed scaffold after one day, respectively. Due to the smaller pore size and high surface area-to-volume ratio, electrospun nanofibers web intercalated in the 3D strands scaffold can act as the cell entrapment systems to increase cell initial attachment efficiency. As shown in Fig. 4a, the seeded hASCs were fully distributed on the nabofibers web between the microfibers layers. On the contrary, there were only a small quantity of cells spread on the printed scaffold (see Fig. 4b), and most cells went through the pores and accumulated at the bottom of the interconnected pores during the cell seeding process. The results indicate that the electrospun nanofibers webs in the hierarchical scaffold can provide a good matrix to improve the attachment efficiency of the seeded cells for tissue regeneration.

The cells proliferation, morphology and interaction on hierarchical scaffold and printed scaffold after $7 \mathrm{~d}$ culture were examined by FE-SEM and the results are shown in Fig. 5. The image of the cell/scaffold showed that most cells were separated on the nanofibers web and secreted abundant ECM (see Fig. 5a). Moreover, a lot cells began to bridge themselves together and occupied a larger surface area in the regular pore structure of 3D strands scaffold. It means that the nanofibers webs in the 3D scaffolds can improve the cell proliferation and accelerate the cells bridge together. However, few cells adhere on the surface of microfibers which also is shown in the $3 \mathrm{D}$ bioprinting scaffold (see Fig. 5b). As shown in Fig. 5b, only a small amount of cells adhere on the surface of strands and the inside wall of the pores with the spherical appearance. We estimate that this phenomenon occurs due to the bigger diameters of printed strands, smooth surfaces of strands and large pore size. The surface topographical and pore structure may go against the cell initial attachment and proliferation which may affect cell seeding efficiency.

Figure 6 shows that the distribution of cells indicates the distance of $60 \mu \mathrm{m}$ from the bottom of the hierarchical scaffold after $21 \mathrm{~d}$ cell culture. The cells infiltrate through the electrospun web and penetrate within the hierarchical scaffold are the very important parameters for designing scaffolds, and the pore size of nanofibers web can be controlled by controlling the deposition time. As shown in Fig. 6, the embedded cells can penetrate and proliferate into the hierarchical scaffold in which PCL web is layered electrospun (deposition time of $3 \mathrm{~s}$ ) after a period of incubation. 

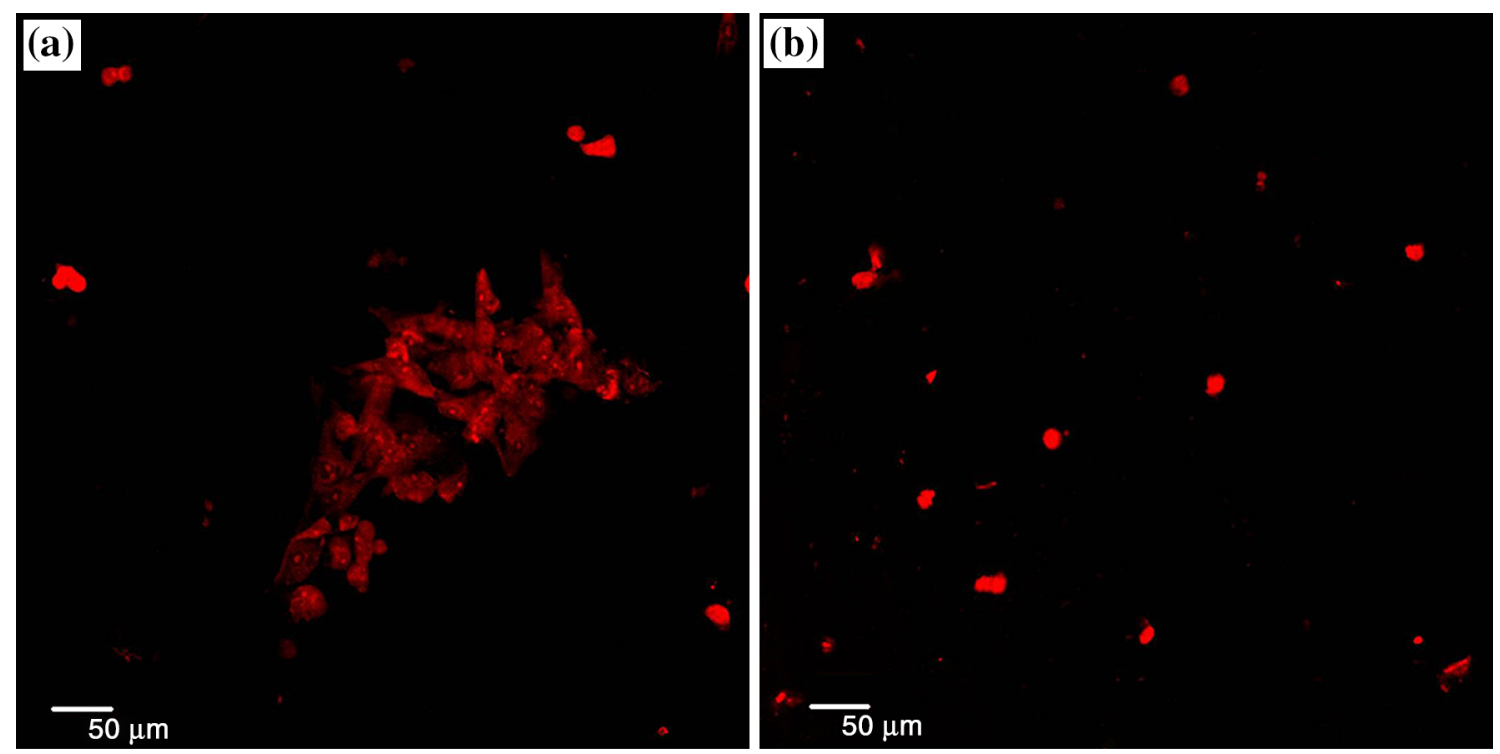

Fig. 4 Micrographs of the initial attachment (1 d) of hASCs: a hierarchical PCL/gel scaffold; b normally bioprinted gel scaffold
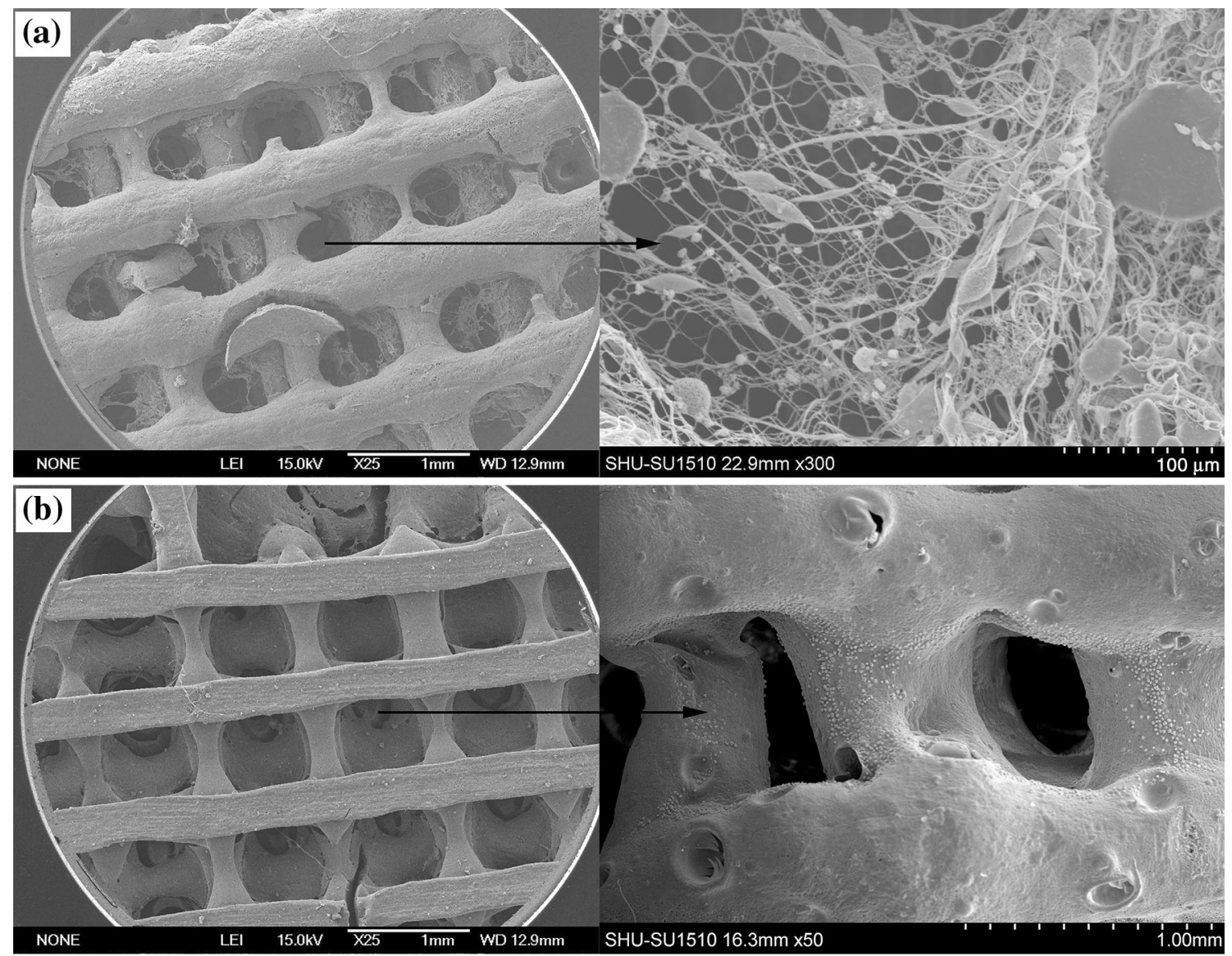

Fig. 5 SEM micrographs of the hASCs cultured on the scaffolds (7 d): a hierarchical PCL/gel scaffold; b normally bioprinted gel scaffold

To evaluate the effects of the hierarchical scaffold on cells proliferation, hASCs were seeded onto the bioprinting and hierarchical scaffolds. As shown in Fig. 7, the initial cell attachment and proliferation of ADCs on the scaffolds were measured by DNA assay using the Hoechst 33258 dye. The initial attachment ( $1 \mathrm{~d})$ of hASCs in the hierarchical scaffold 


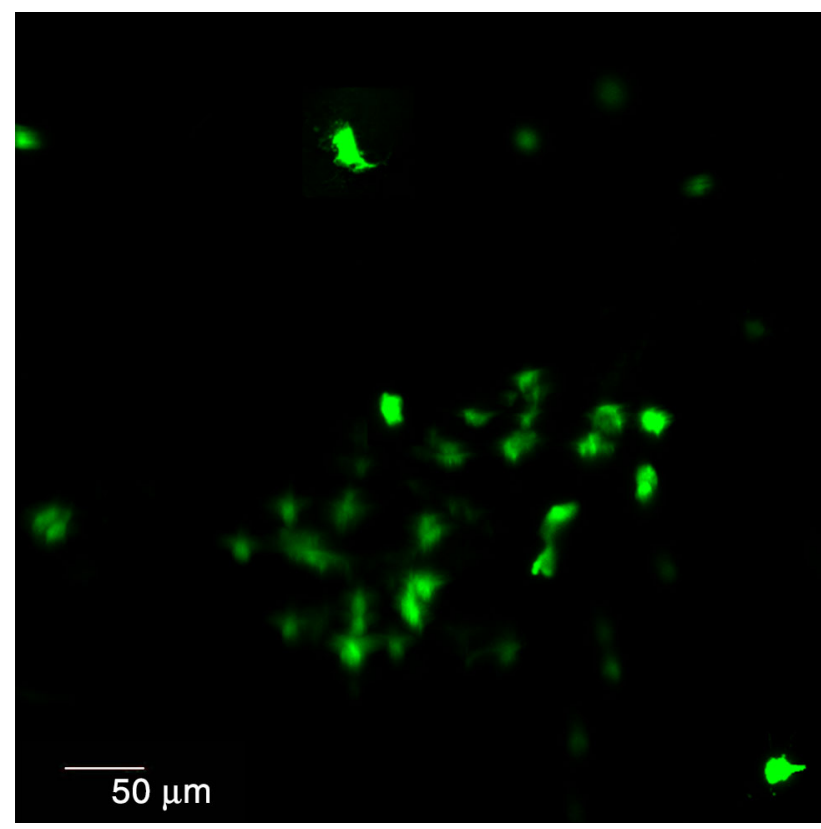

Fig. 6 Micrograph of the distribution of hASCs inside the scaffold ( $21 \mathrm{~d}$, up from the bottom of scaffold $60 \mu \mathrm{m}$ )

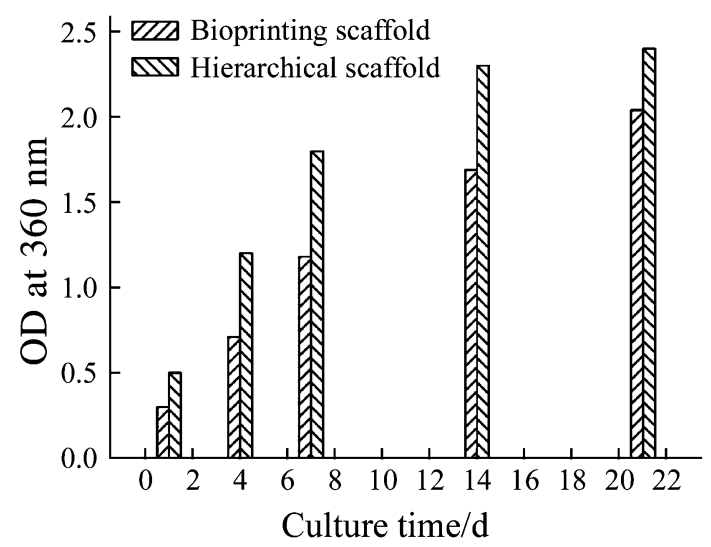

Fig. 7 Cell attachment and proliferation of hASCs on the bioprinting and hierarchical scaffolds by DNA assay using Hoechst 33258 dye

was certainly high relative to the bioprinting one. The reason was that the nanofibers web in the hierarchical scaffold could provide a matrix to maintain the embedded cells. Along with the cultivation times, a significant increment of cell proliferation was observed on the hierarchical scaffolds compared to the printed ones, which likely due to the nanofibers in the hierarchical scaffolds afforded the higher surface area-tovolume ratio to enhance the cell adhesion and proliferation. However, around $14 \mathrm{~d}$ and $21 \mathrm{~d}$ of the culture, no significant increment on the hierarchical scaffold was found because of the saturation of the cell proliferation.

From the results, we can come to the conclusion that the hierarchical scaffold with precisely controlled of 3D porous architecture and nanofibers nonwovens can provide an ideal matrix for attachment, growth and proliferation of seeded cells compared to the 3D bioprinting scaffold. The hierarchical PCL/gel scaffold fabricated by combined 3D bioprinting and electrospinning can enhance cell attachment, growth and proliferation to promote rapid and stable tissue regeneration.

\section{Conclusions}

In the present research, hierarchical 3D scaffolds have been prepared via combination of 3D bioprinting and electrospinning from gel biomaterials and PCL solution for tissue engineering applications. Scaffolds characterizations and cell culture analysis are performed to explore the effects of hierarchical scaffold on the interactions between cells and scaffold. Using the compound technology, a hierarchical scaffold composed of gel microfibers forming the controlled 3D structure with interconnected macropores network and electrospun nanofibers web with micropores and high surface area can be successfully fabricated. The results of cell culture show that the hierarchical scaffold can improve initial cell attachment, cells growth and proliferation compared to the 3D bioprinting scaffold. The hierarchical PCL/gel scaffold has demonstrated the potential of combination of 3D bioprinting and electrospinning for scaffold fabrication in tissue engineering.

Acknowledgments This research was supported by the National Natural Science Foundation of China (Grant No. 51375292).

\section{References}

1. Vacanti JP, Morse MA, Saltzman WM (1988) Selective cell transplantation using bioabsorbable artificial polymers as matrices. J Pediatr Surg 23:3-9

2. Langer R, Vacanti JP (1993) Tissue engineering. Science 260:920-926

3. Wang DL (2011) Stem cells tissue engineering technology. Sci. Pr., Beijing

4. Wang SG (2001) Cells scaffold of tissue engineering and the related technologies. Modern Rehabil. 5(16-17):41

5. Shuai CJ, Mao ZZ, Lu HB, Nie Y, Hu HL, Peng SP (2013) Fabrication of porous polyvinyl alcohol scaffold for bone tissue engineering via selective laser sintering. Biofabrication 5:015014

6. Poot AA, Grijpma DW, Feijen J, Vermes I (2012) Vascular tissue engineering. Ned Tijdschr Klin Chem Labgeneesk 37:44-46

7. Carfi PF, Rigogliuso S, La Carrubba V, Mannella GA, Ghersi G, Brucato V (2012) Poly lactic acid based scaffolds for vascular tissue engineering. Chem Eng Trans 27:409-414

8. Teixeira AI, Abrams GA, Bertics PJ, Murphy CJ, Nealey PF (2003) Epithelial contact guidance on well-defined micro-and nanostructured substrates. J Cell Sci 116:1881-1892

9. Park SA, Kim HJ, Lee SH, Lee JH, Kim HK, Yoon TR (2011) Fabrication of nano/microfiber scaffolds using a combination of rapid prototyping and electrospinning systems. Polym Eng Sci $51: 1883-1890$ 
10. Gautam S, Dinda AK, Mishra NC (2013) Fabrication and characterization of PCL/gelatin composite nanofibrous scaffold for tissue engineering applications by electrospinning method. Mater Sci Eng 33:1228-1235

11. Duan B, Cheung WL, Wang M (2011) Optimized fabrication of Ca-P/PHBV nanocomposite scaffolds via selective laser sintering for bone tissue engineering. Biofabrication 3:015001

12. Melchels FPW, Domingos MAN, Klein TJ, Malda J, Bartolo PJ, Hutmacher DW (2012) Additive manufacturing of tissues and organs. Prog Polym Sci 37:1079-1104

13. Nair K, Gandhi M, Khalil S, Yan KC, Marcolongo M, Barbee K, Sun W (2009) Characterization of cell viability during bioprinting processes. Biotechnol J 4:1168-1177

14. Seyednejad H, Gawlitta D, Kuiper RV, Bruin AD, Nostrum CF, Vermonden T, Dhert WJA, Hennink WE (2012) In vivo biocompatibility and biodegradation adation of 3D-printed porous scaffolds based on a hydroxyl-functionalized poly ( $\varepsilon$-caprolactone). Biomaterials 33:4309-4318

15. Dombrowski F, Caso PWG, Laschke MW, Klein M, Gunster J, Berger G (2013) 3-D printed bioactive bone replacement scaffolds of alkaline substituted ortho-phosphates containing metaand di-phosphates. Key Eng Mater 529-530:138-142

16. Ibrahim TO, Yin Y (2013) Bioprinting toward organ fabrication: challenges and future trends. IEEE Trans Biomed Eng 60:691-699

17. Liu HX, Yan YN, Wang XH, Xiong Z, Cheng J (2005) Threedimensional controlled assembling of hepatocytes. J Tsinghua Univ (Sci \& Tech) 45: 1009-1011, 1031

18. Landers R, Hubnerb U, Schmelzeisenb R (2002) Rapid prototyping of scaffolds derived from thermoreversible hydrogels and tailored for applications in tissue engineering. Biomaterials 23:4437-4447

19. Liu F, Zhang RJ, Yan YN, Liu HX (2006) Rapid prototyping of gelatin/sodium alginate tissue engineering scaffolds. J Tsinghua Univ (Sci \& Tech) 46:1357-1360

20. Eladale T, Bard J (1972) Collagen substrata for studies on cell behavior. J Cell Biol 54:626-637
21. Colon G, Ward BC, Matster TJ (2006) Increased osteoblast and decreased staphylococcus epidermis functions on nanophase $\mathrm{ZnO}$ and $\mathrm{TiO}_{2}$. J Biomed Mater Res A 78:595-604

22. Ye L, Zeng XC, Li HJ, Ai Y (2010) Fabrication and biocompatibility of nano non-stoichiometric apatite and poly (epsiloncaprolactone) composite scaffold by using prototyping controlled process. J Mater Sci 21:753-760

23. Baker BM, Handorf AM, Ionescu LC, Li WJ, Mauck RL (2009) New directions in nanofibrous scaffolds for soft tissue engineering and regeneration. Expert Rev Med Devices 6:515-532

24. Xu H, Cui WG, Chang J (2013) Fabrication of patterned PDLLA/ PCL composite scaffold by electrospinning. J Appl Polym Sci 127:1550-1554

25. Kim GM, Le KHT, Giannitelli SM, Lee YJ, Rainer A, Trombetta M (2013) Electrospinning of PCL/PVP blends for tissue engineering scaffolds. J Mater Sci 24:1425-1442

26. Liu DL, Liu YY, Wang QG, Jing CJ, Hu QX (2012) Multiphysics coupling analysis and experiment of low-temperature deposition manufacturing and electrospinning for multi-scale tissue engineering scaffold. J Mech Eng 48:137-143

27. Ramay HR, Zhang M (2003) Preparation of porous hydroxyapatite scaffolds by combination of the gel-casting and polymer sponge methods. Biomaterials 24:3293-3302

28. Mikos AG, Thorsen AJ, Czerwonka LA (1994) Preparation and characterization of poly (L-lactic acid) foams. Polymer 35: 1068-1077

29. Stoppato M, Stevens HY, Carletti E, Migliaresi C, Motta A, Guldberg RE (2013) Effects of silk fibroin fiber incorporation on mechanical properties, endothelial cell colonization and vascularization of PDLLA scaffolds. Biomaterials 34:4573-4581

30. Matsuzaka K, Walboomers XF, Ruijter JE (1999) The effects of poly-lactic acid with parallel surface micro groove on osteoblast growth factor. Biomaterials 20:1293-1301

31. Nam YS, Yoon JJ, Park TG (2000) A novel fabrication method of macroporous biodegradable polymer scaffolds using gas forming salt as a porogen additive. J. Biomed Mater Res Part B 53:1-7 